Gynecologic and

Obstetric Investigation
Gynecol Obstet Invest 2015;79:1-12

DOI: $10.1159 / 000362231$
Received: January 15, 2014

Accepted after revision: March 14, 2014

Published online: May 16, 2014

\title{
The Role of Acupuncture in in vitro Fertilization: A Systematic Review and Meta-Analysis
}

\author{
Chunjuan Shen $^{a} \quad$ Manxiang Wu ${ }^{b}$ Defeng Shu ${ }^{a}$ Xiaojie Zhao ${ }^{a}$ Ying Gao $^{a}$ \\ ${ }^{a}$ Department of Obstetrics and Gynecology, and ${ }^{b}$ Institute of Integrated Traditional Chinese and Western Medicine, \\ Union Hospital, Tongji Medical College, Huazhong University of Science and Technology, Wuhan, China
}

\section{Key Words}

Acupuncture $\cdot$ In vitro fertilization $\cdot$ Clinical pregnancy rate . Embryo transfer $\cdot$ Systematic review

\begin{abstract}
Background/Aims: In recent years, acupuncture has become more and more popular in the management of subfertility. The aim of this study was to evaluate the impact of acupuncture during in vitro fertilization (IVF) treatment on the outcomes of clinical pregnancy in published randomized studies. Methods: This is a systematic review and meta-analysis. Data sources used were MEDLINE, Embase, Web of Knowledge and the Chinese Biomedical Database. Results: There was no statistically significant difference between the acupuncture group and no acupuncture (intervention) controls around the time of embryo transfer (ET; risk ratio, RR, 1.24, $95 \%$ confidence interval, $\mathrm{Cl}, 1.02-1.50$ ) or in unblinded trials, trials blinded to physicians and double-blind trials $(95 \% \mathrm{Cl}$ $1.26-1.88,0.82-1.33$ and $0.89-1.25$, respectively). This was also the case when comparing acupuncture with sham acupuncture controls around the time of ET (RR, $1.03,95 \% \mathrm{Cl}$ $0.87-1.22$ ) or when restricting to unblinded trials, trials blinded to physicians and double-blind trials $(95 \% \mathrm{Cl} 0.80-2.02$, $0.82-1.18$ and $0.77-1.17$, respectively). There was a statistically significant difference when performed at $30 \mathrm{~min}$ after ET and implantation phase (RR 1.76, 95\% Cl 1.22-2.55). There was also a statistically significant difference when performed
\end{abstract}

at follicle phase and $25 \mathrm{~min}$ before and after ET (RR 1.56, 95\% Cl 1.04-2.33). Conclusion: Our study showed that acupuncture did not significantly improve the IVF clinical pregnancy rate when performed only at the time of ET, while we found pooled benefit of acupuncture for IVF when performed at follicle phase and $25 \mathrm{~min}$ before and after ET, as well as $30 \mathrm{~min}$ after ET and implantation phase.

(c) 2014 S. Karger AG, Base

\section{Introduction}

Over the past decade, much research has been devoted to increasing the in vitro fertilization (IVF) success rate, improving the quality of embryos, modulating the hormones, and managing the environment of the uterus. However, the pregnancy rate success remains low, so more and more couples affected by infertility try to seek other fertility cures. Acupuncture is an important part of traditional Chinese medicine and dates back at least 3,000 years. In recent years, acupuncture is more and more popular in the management of subfertility, but its effectiveness has remained controversial [1]. Some studies showed that acupuncture was significant in improving IVF outcomes [24]. The specific rationales were displayed as follows. First, psychological and environmental factors among Chinese are associated with early pregnancy [5]. The acupuncture regimen may abate stress around embryo transfer (ET)

\section{KARGER 125}

C 2014 S. Karger AG, Base

0378-7346/14/0791-0001\$39.50/0

E-Mail karger@karger.com

www.karger.com/goi
Ying Gao

Department of Obstetrics and Gynecology, Union Hospital

Tongji Medical College, Huazhong University of Science and Technology

Wuhan, Hubei 430022 (China)

E-Mail gying13@163.com 
and possibly improve pregnancy rates [6]. Second, acupuncture may enhance blood flow of the uterus and reduce uterine artery blood flow impedance [7]. Third, acupuncture treatment may enhance the hormonal balance of the embryo implantation stage of IVF in the treatment of infertility [4]. Another study also showed that acupuncture may promote the release of neurotransmitters, thereby mediating the development of hormones [8]. Fourth, acupuncture may regulate the levels of stem cell factor in follicular fluid and serum and improve oocyte quality [3]. However, the outcomes of recent studies have been adverse, and showed that the role of acupuncture in the IVF process was negligible or even nonexistent [9-11].

Many recent systematic reviews analyzed the effects of acupuncture among women undergoing IVF [1, 12-15]. These studies concluded conflicting results to different subgroups. We conducted a systematic review and metaanalysis of randomized controlled trials (RCTs) involving different time points of acupuncture during IVF treatment to generate a more precise estimate of the effect of acupuncture on IVF outcome.

\section{Materials and Methods}

\section{Literature Search}

We searched PubMed (1977 to September 2013), Embase (1974 to September 2013), Web of knowledge (Science) and the Chinese Biomedical Database. Medical subject headings (MeSH) included studies of acupuncture ('acupuncture', 'moxibustion' and 'electroacupuncture') and other studies of IVF and intracytoplasmic sperm injection (ICSI; 'in vitro fertilization', 'assisted reproduction', 'infertility' and 'embryo transfer'). We used 'AND' to connect the two parts.

In the Chinese Biomedical Database, we searched as follows: ZHEN JIU (which means 'acupuncture'), 'ZHEN CI' ('acupuncture'), 'TI WAI SHOU JING' ('in vitro fertilization'), 'FU ZHU SHENG ZHI JI SHU' ('assisted reproduction technology') and 'PEI TAI YI ZHI' ('embryo transfer').

\section{Study Selection}

First, we selected the RCTs. Second, the target population was women undergoing IVF with or without ICSI. IVF patients with a tendency for poorer prognosis were excluded. Women who began the IVF process but did not undergo ET were considered. This preserved the groups created by the randomization and reduced the chance of a type I error [13]. Third, some methods of acupuncture (needle, laser or electro) were accepted and compared with the no or sham (placebo) acupuncture group. In the acupuncture group, acupuncture could be needled into the traditional meridian points. The intervention was performed during the IVF or ICSI cycle. Fourth, any type of publication such as an abstract and full article was accepted, as well as any language of publication.

The acupuncture points used may be different but were chosen according to the principles of traditional Chinese medicine. Some studies only included body acupuncture (even the body acupuncture points were variable), while some studies included body and ear acupuncture.

Data concerning the ongoing pregnancy rate and the live birth rate were not always integrated from these included trials. The clinical pregnancy rate (CPR) that almost all trials reported from a single IVF cycle per woman randomized to acupuncture or control intervention was more accurate than the chemical pregnancy rate. Therefore, we picked CPR to represent the effect of acupuncture on IVF. Clinical pregnancy was defined as the ultrasound identification of an intrauterine gestational sac after IVF treatment. Exclusion criteria: Retrospective studies, studies with a crossover design and studies with an obscure description of the CPR were excluded. Of course trials with no exact numbers of pregnancies and initials of all patients were also not considered. Since pain experience varies from individual to individual, the optimal method of conscious sedation may also be individualized [16]. Those studies that performed during oocyte retrieval to relieve pain were excluded.

\section{Data Extraction}

Two authors independently selected articles and extracted data. Data extracted included quality of the methods, participants, interventions and outcomes. Allocation concealment, blinding, sample size, population, protocol of intervention, type of control (no or sham acupuncture) and pregnancy outcomes were listed from the full-text articles and abstracts. The two authors discussed disagreement or contacted the corresponding authors with regard to the design and outcomes of their trials in order to clarify any ambiguities.

\section{Data Synthesis and Analysis}

The results were pooled and expressed as relative risks (RR) with $95 \%$ confidence intervals (CI). The heterogeneity of treatment effects was evaluated using a forest plot and statistically using $\mathrm{I}^{2}$. If the $\mathrm{I}^{2}$ was higher than $50 \%$, the random effect model was applied. In this paper, the type of control, acupuncture time and blindedness were used for sensitivity subgroup analyses.

We defined 3 broad groups of studies according to the different time points of acupuncture. The three time points were as follows: (1) 25 min before and after ET (around ET), (2) follicle phase and $25 \mathrm{~min}$ before and after ET and (3) $30 \mathrm{~min}$ after ET and implantation phase. We analyzed the sham-controlled and no interventioncontrolled trials separately. Further, the double-blind, single-blind and unblinded trials were analyzed separately in the group around ET. The other 2 groups did not have enough trials, so we only analyzed the acupuncture and control trials.

\section{Results}

Overall, 21 RCTs with a total of 5,428 participants were contained in our review; 14 of the 21 included trials ( $\mathrm{n}=$ 3,971 ) met standards when acupuncture was performed around the time of ET, while the remaining 8 trials $(\mathrm{n}=$ 1,457) fulfilled the criteria when acupuncture was performed before/after and around ET. See figures 1-4 for meta-analyses of all studies. 
No intervention control group

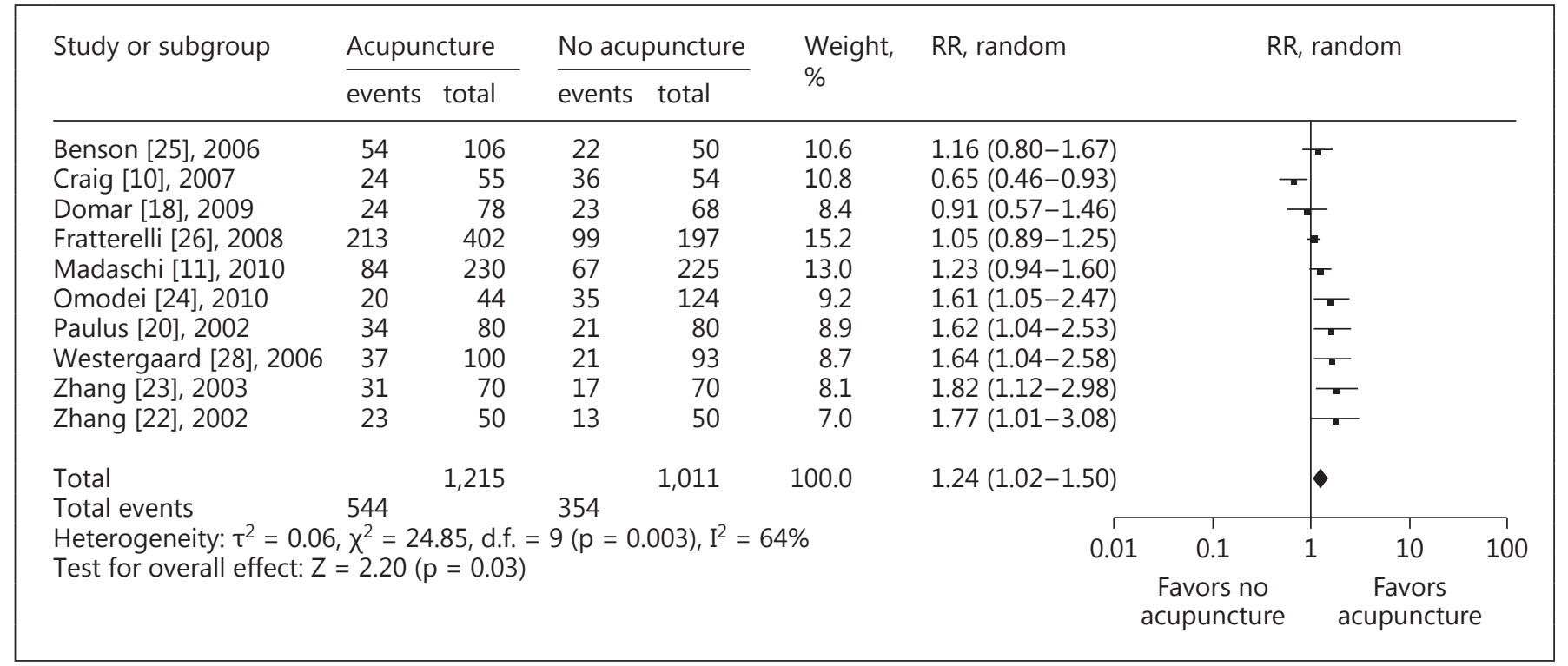

Unblinded: no intervention control group

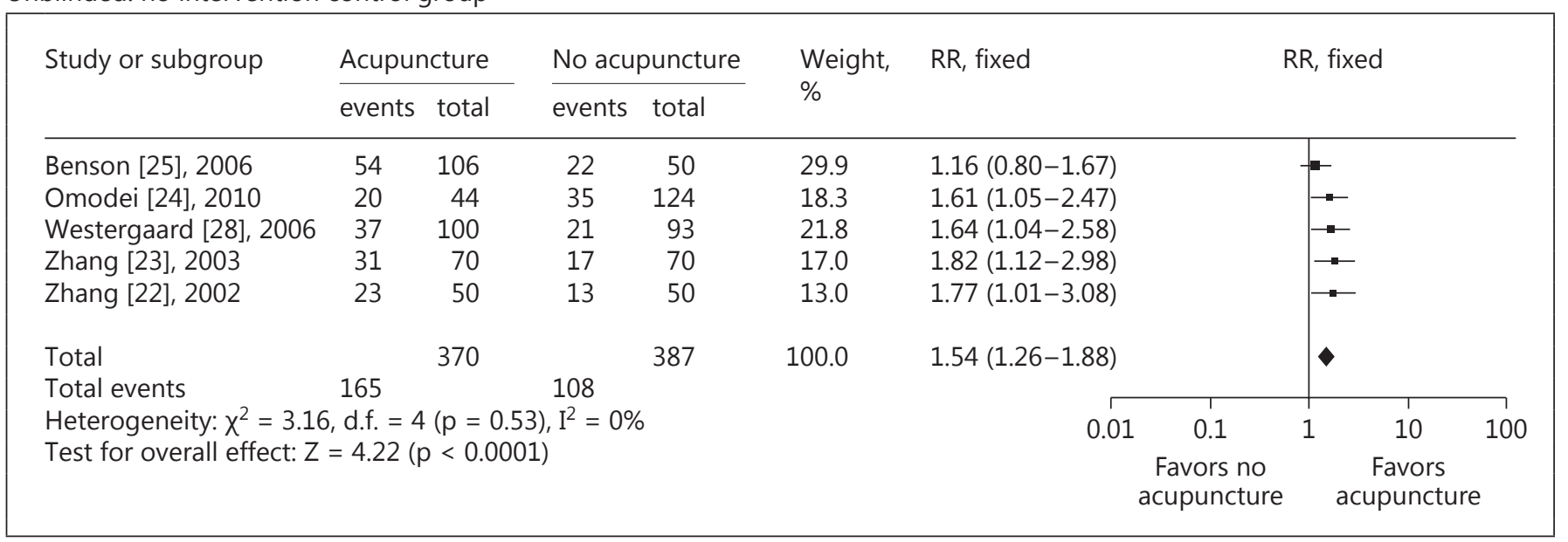

Blinded to physicians: no intervention control group

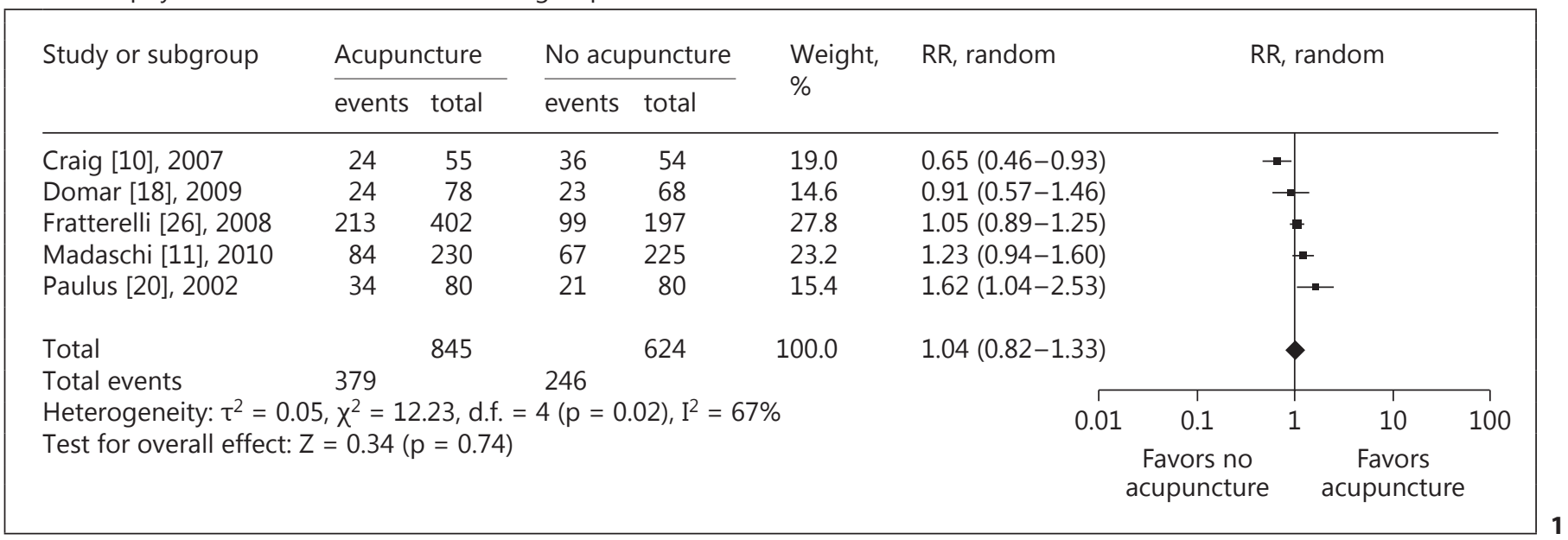

(For legend see next page.)

The Role of Acupuncture in in vitro Fertilization
Gynecol Obstet Invest 2015;79:1-12 DOI: $10.1159 / 000362231$ 
Double-blind: no intervention control group

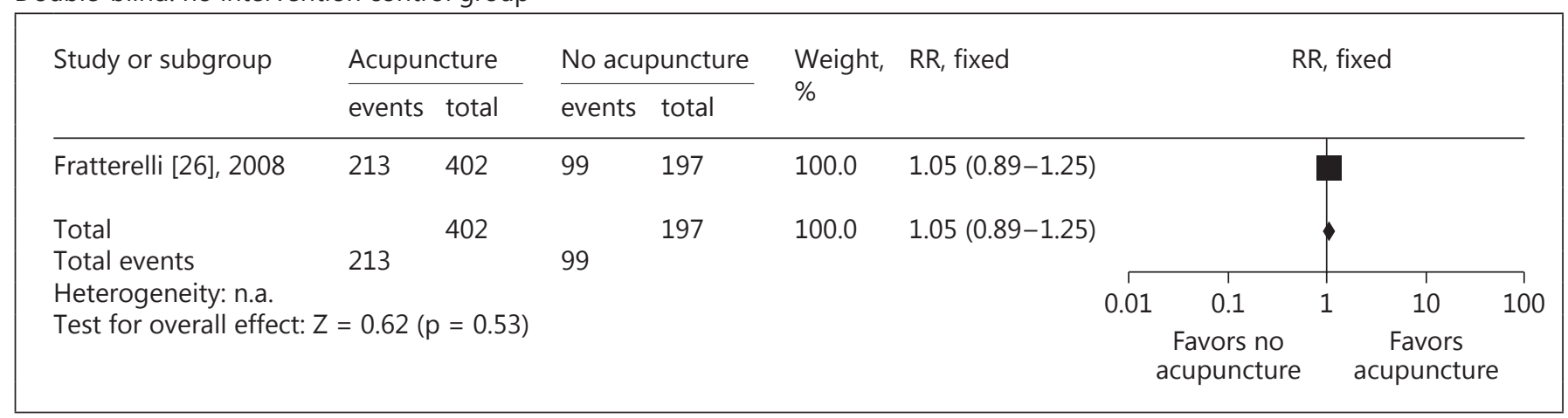

Fig. 1. Meta-analysis of the studies evaluating the effect of acupuncture administered around the time of ET on the CPR in women undergoing IVF in the no intervention control groups. Values in parentheses are $95 \%$ CI. RR: Mantel-Haenszel method. n.a. = Not applicable.

\section{Acupuncture Only around the Time of ET}

In the study of Westergaard et al. [28], there were 2 acupuncture groups: ACU1 (patients who received acupuncture on the day of ET) and ACU2 (those who received acupuncture on the day of ET and again 2 days later). In this case, we put the ACU1 patients into the group of acupuncture around ET and the ACU2 subjects into the group of acupuncture after and around ET. There were 14 RCTs $(n=3,971)$ performing only around the time of ET. The quality of the included trials is summarized in table 1; 7 of the 14 studies were published as full reports [17-23], while the remaining 7 were published as conference abstracts $[10,11,24-28] ; 4$ of the studies were in Europe (2 in Denmark [17, 18], 1 in Brazil [11], 1 in Germany [20]), 3 in China [21-23] and 7 in the USA [10, $18,19,24-27]$.

Of these trials, 2 included women with good quality embryos [20,27], while the other trials included women without clear quality embryos; in 1 trial [11] all participants underwent ICSI, and not IVF, but all other trials reported using ICSI or IVF for participants - 10 trials included acupuncture and a no intervention control group. There were 4 unblinded trials [22, 24, 25, 28]. For 5 trials, the ET physicians were blinded to the treatment assignments $[10,11,18,20,26]$. For 1 trial, the ET physicians and patients were double blinded to the treatment assignments [26]. All studies used traditional needle acupuncture, and none used electroacupuncture. In addition, the study of Benson et al. [25] and Fratterelli et al. [26] included 5-arm groups (acupuncture, laser acupuncture, sham laser acupuncture, relax, no intervention), in which laser acupuncture was in 2 of the 5 arms of the trials. The study of Zhang et al. [23] contained 3 groups (acupuncture, no acupuncture, sham acupuncture).

Of the 14 trials, 7 performed sham (placebo) acupuncture in the control group; 2 trials $[23,25]$ belonged to the unblinded trials group and $5[17,19,21,26,27]$ belonged to the group of trials that were blinded to the physicians. Of the above 5 trials, all except the study of Paulus et al. [27] were double-blind trials. The methods of sham acupuncture were practiced in various ways: using superficial needling and blunt (placebo) needles on the true acupuncture points, using acupuncture on other places such as thighs and arms, or using laser sham acupuncture. But the principles of Chinese medicine implied in the sham acupuncture treatment had no influence on human fertility. In all trials, women received acupuncture on the day of ET, 25 min before and after the ET procedure.

In these trials there was similarity between the acupuncture group and the control group except for the study of Madaschi et al. [11], in which age was the essential factor in classifying the patients and some randomized women did not complete the treatment in the IVF process (i.e. no ET).

\section{Acupuncture at Follicle Phase and $25 \mathrm{~min}$ before and after ET}

A total of 4 trials were included in the subgroup. The characteristics of the included trials are summarized in table 2. Acupuncture at follicle phase only was used in 2 of the trials $[29,30]$, while the other 2 trials used acupuncture at follicle phase and around ET [31, 32]. All 4 trials were published as full reports - 1 in the UK [31], 1 in Australia [32] and 2 in China [29, 30]. 
Sham acupuncture group

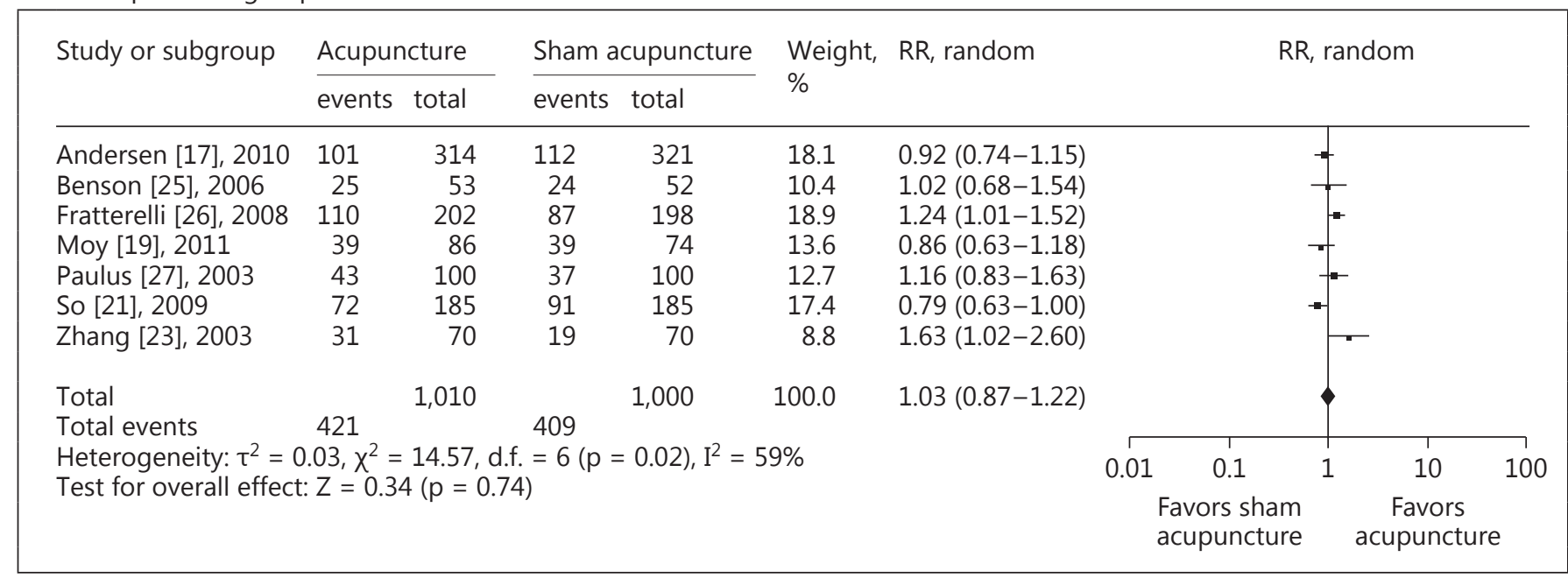

Unblinded: sham acupuncture group

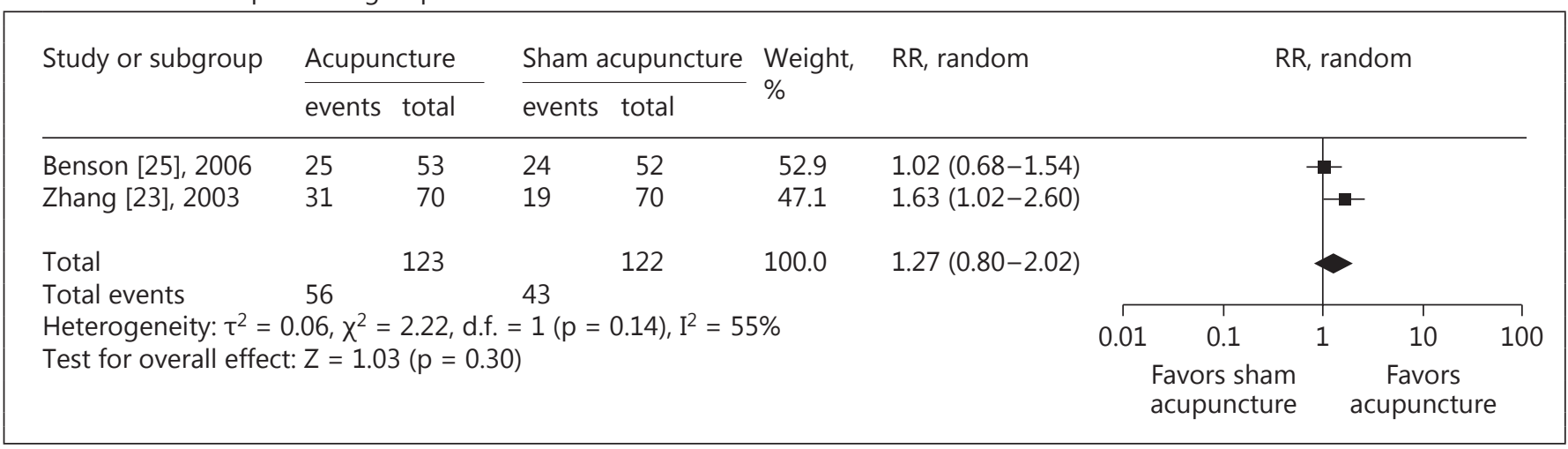

Blinded to physicians: sham acupuncture group

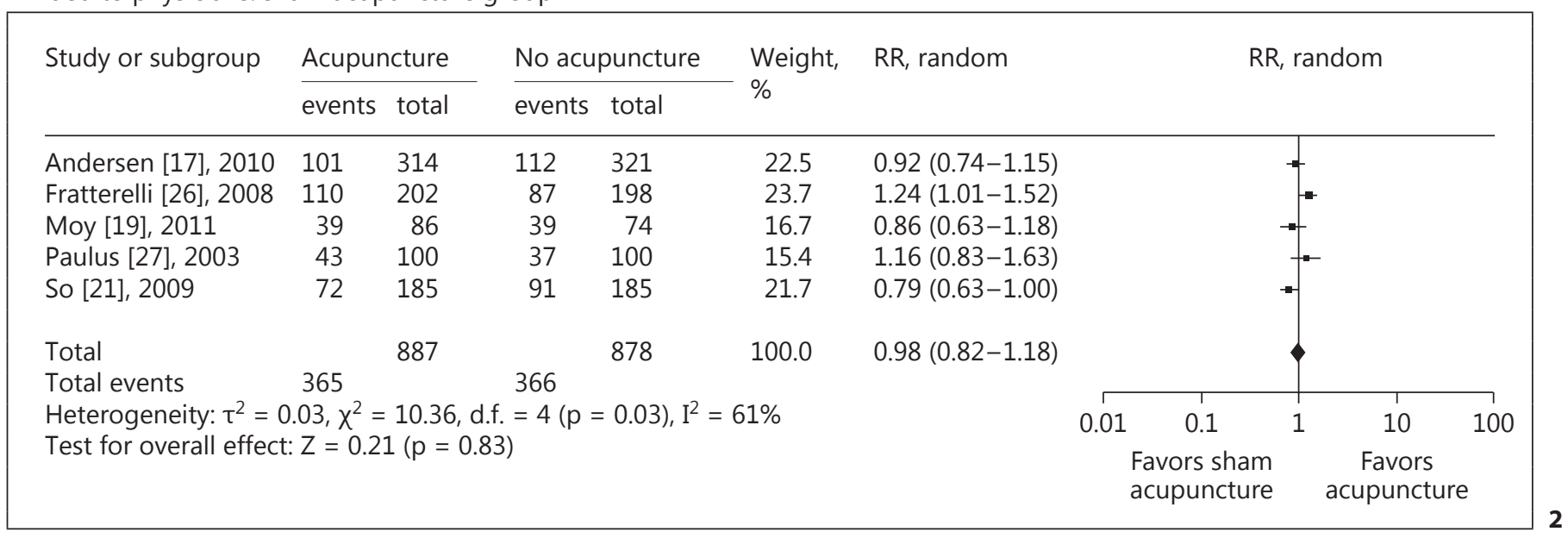

(For legend see next page.)

The Role of Acupuncture in in vitro Fertilization 
Double-blind: sham acupuncture group

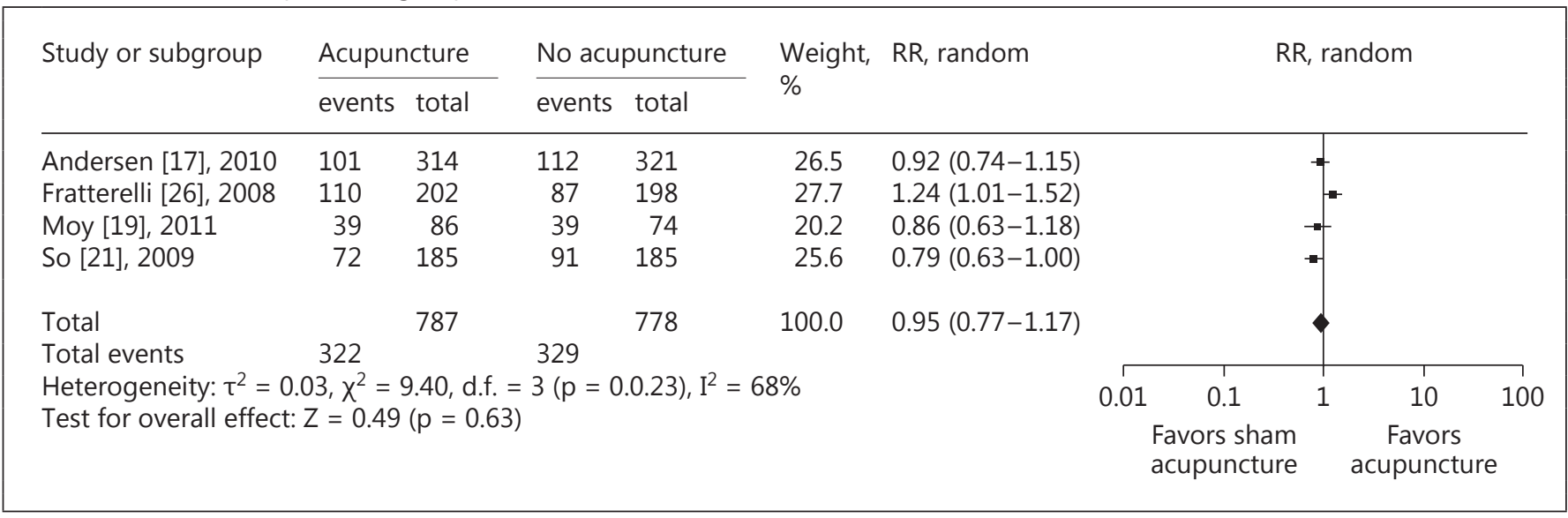

Fig. 2. Meta-analysis of the studies evaluating the effect of acupuncture administered around the time of ET on the CPR in women undergoing IVF in sham control groups. Values in parentheses are 95\% CI. RR: MantelHaenszel method.

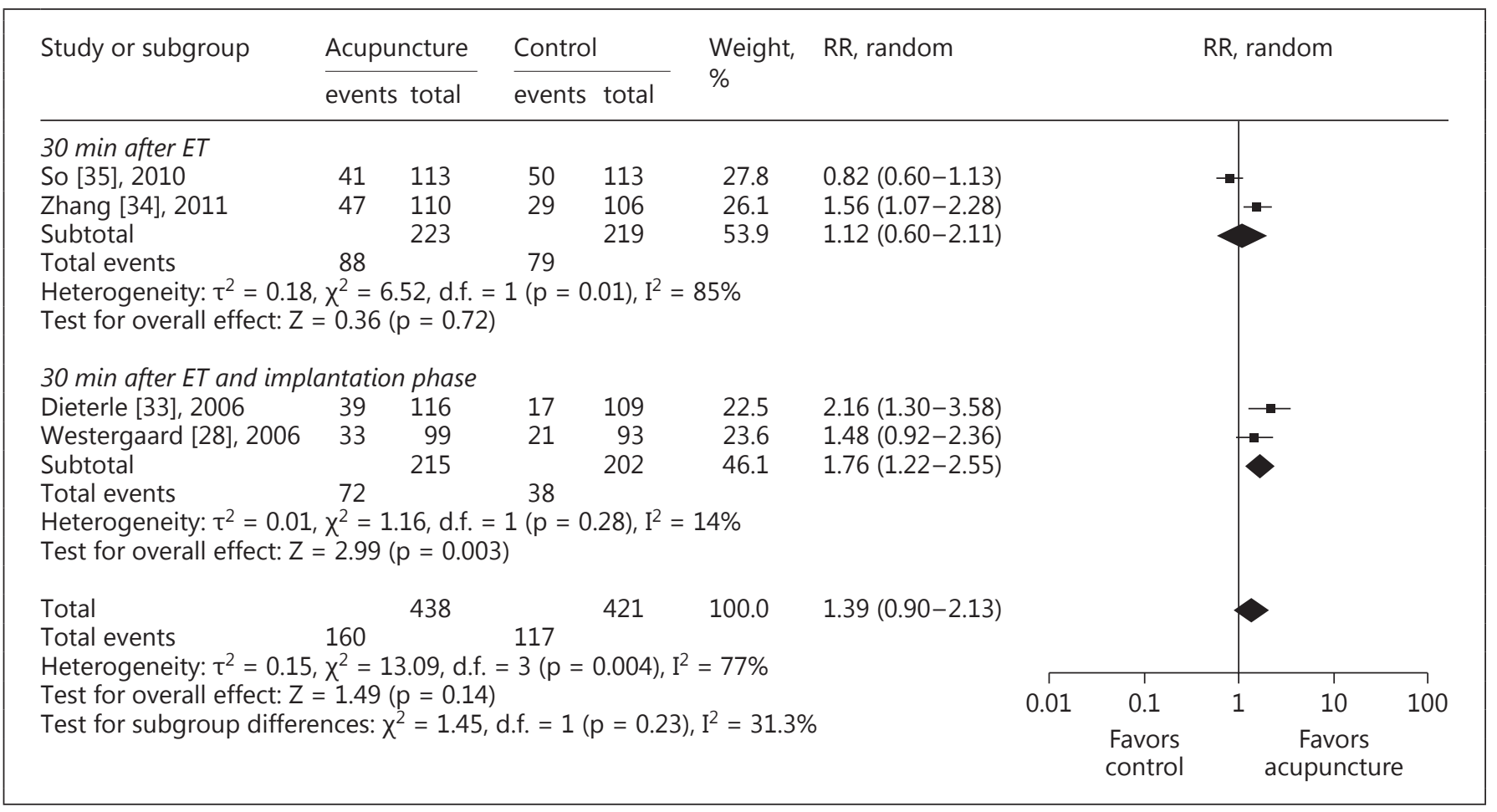

Fig. 3. Meta-analysis of the studies evaluating the effect of acupuncture administered at 30 min after ET and implantation phase on the CPR in women undergoing IVF in placebo-controlled trials. Values in parentheses are 95\% CI. RR: Mantel-Haenszel method. 


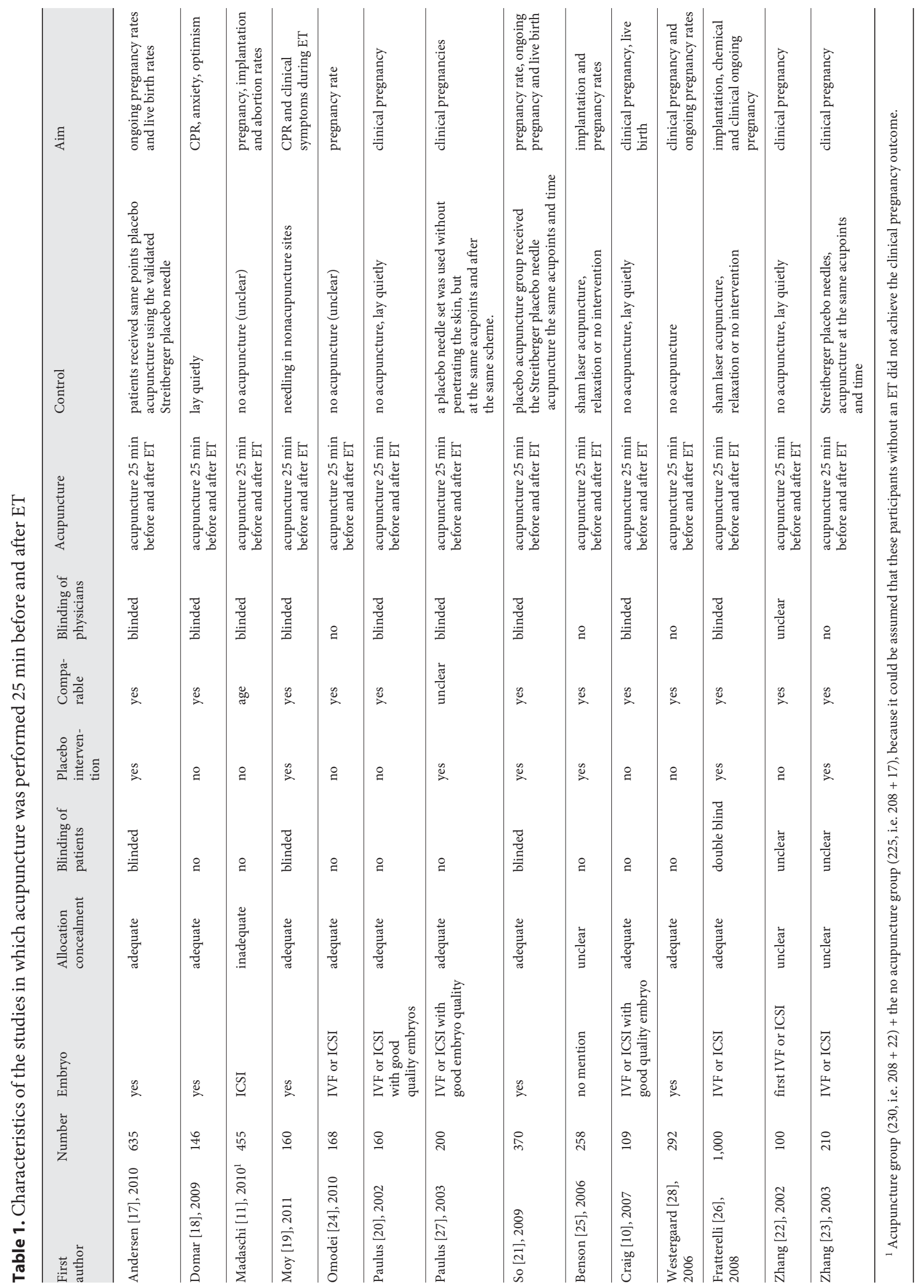




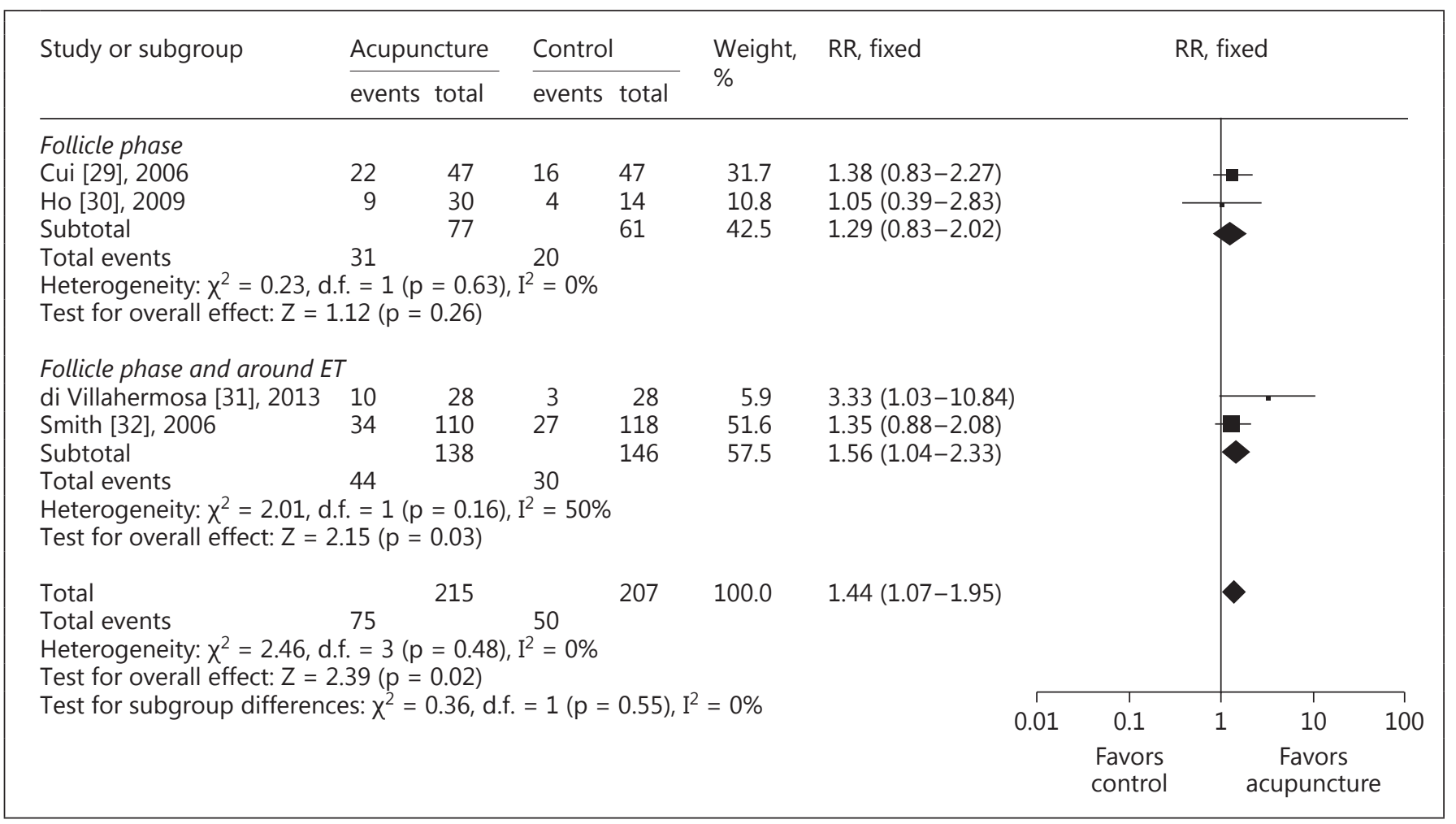

Fig. 4. Meta-analysis of the studies evaluating the effect of acupuncture administered at follicle phase and $25 \mathrm{~min}$ before and after ET on the CPR in women undergoing IVF. Values in parentheses are 95\% CI. RR: MantelHaenszel method.

\section{Acupuncture at 30 min after ET and Implantation \\ Phase}

In all, 4 trials $[28,33-35]$ were included when acupuncture was performed after and around the time of ET (1 in Germany [33], 1 in Denmark [28] and 2 in China $[34,35])$. The characteristics of the included trials are summarized in table 2. The study of Zhang et al. [34] included 2 groups (mock transcutaneous electrical acupoint stimulation treatment: $30 \mathrm{~min}$ after ET; single transcutaneous electrical acupoint stimulation treatment: $30 \mathrm{~min}$ after ET). In the study of Westergaard et al. [28], there were 2 acupuncture groups: ACU1 (patients who received acupuncture on the day of ET) and ACU2 (those who received acupuncture on the day of ET and again 2 days later). In this case we put the ACU1 patients into the group of acupuncture around ET and the ACU2 subjects into the group of acupuncture after and around ET. The studies of So et al. [35] and Zhang et al. [34] used electroacupuncture in the trials, whereas the others used traditional needle acupuncture.

\section{IVF Treatment Outcome}

There was no statistically significant difference between acupuncture and no acupuncture (intervention) controls around the time of ET (RR 1.24, 95\% CI 1.02$1.50, \mathrm{I}^{2}=64 \%, 10$ trials, 2,226 participants) or when restricting to unblinded trials (RR 1.54, 95\% CI 1.26-1.88, $\mathrm{I}^{2}=0 \%, 5$ trials, 757 participants) or trials blinded to physicians (RR 1.04, 95\% CI 0.82-1.33, $\mathrm{I}^{2}=67 \%, 5$ trials, 1,469 participants) or double-blind trials (RR 1.05, 95\% CI $0.89-1.25, I^{2}=0 \%, 1$ trial, 599 participants; fig. 2). The same was true when comparing acupuncture with sham acupuncture controls around the time of ET (RR 1.03, 95\% CI 0.87-1.22, $\mathrm{I}^{2}=59 \%, 7$ trials, 2,010 participants) or when restricting to unblinded trials (RR $1.27,95 \% \mathrm{CI}$ $0.80-2.02, \mathrm{I}^{2}=55 \%, 2$ trials, 245 participants) or trials blinded to physicians (RR $0.98,95 \%$ CI $0.82-1.18, \mathrm{I}^{2}=$ $61 \%, 5$ trials, 1,765 participants) or double-blind trials (RR 0.95, 95\% CI 0.77-1.17, I ${ }^{2}=68 \%, 4$ trials, 1,565 participants; fig. 3). There was no statistically significant difference between acupuncture and controls when combining all trials performed $30 \mathrm{~min}$ after ET (RR 1.12, 95\% CI 


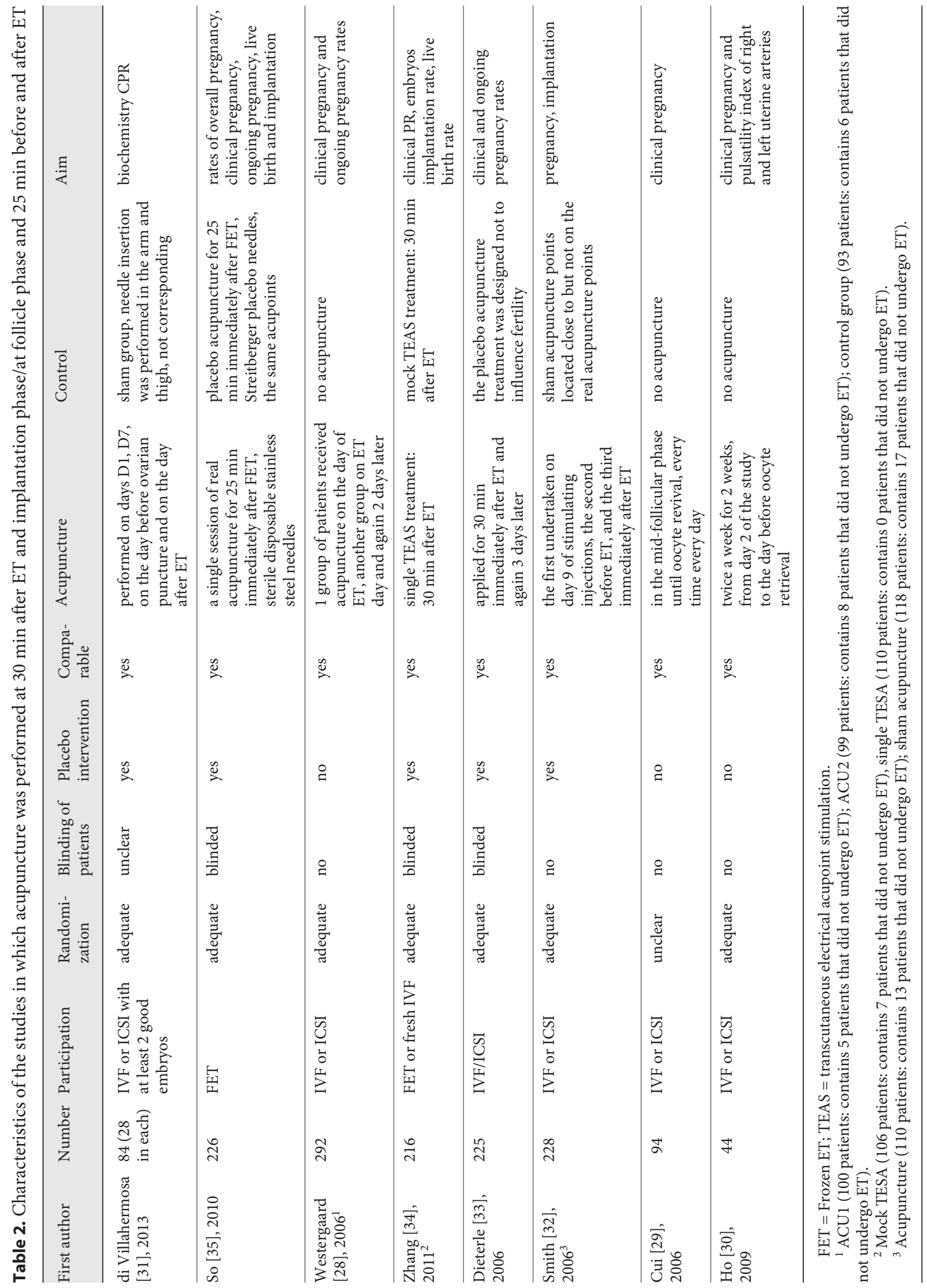


$0.60-2.11, \mathrm{I}^{2}=85 \%, 2$ trials, 442 participants), but there was a statistically significant difference between acupuncture and controls when combining all trials performed 30 min after ET and implantation phase (RR 1.76, 95\% CI $1.22-2.55, \mathrm{I}^{2}=14 \%, 2$ trials, 417 participants). When performing at follicle phase, there was no statistically significant difference between acupuncture and controls (RR $1.29,95 \%$ CI $0.83-2.02, \mathrm{I}^{2}=0 \%, 2$ trials, 138 participants). When performing at follicle phase and around ET, there was also a statistically significant difference between acupuncture and controls (RR 1.56, 95\% CI 1.04-2.33, $\mathrm{I}^{2}=$ $50 \%, 2$ trials, 284 participants).

\section{Discussion}

The outcomes of our review showed no significant improvement in clinical pregnancy when acupuncture was performed around the time of ET. According to our results, in sham-controlled trials, we found no statistically significant pooled benefits of acupuncture administered around the time of ET in IVF; when restricting to unblinded or double-blind trials, there was also no statistically significant improvement in clinical pregnancy. In no treatment-controlled trials, when restricting to unblinded trials, there was a more statistically significant difference. However, when restricting to trials blinded to physicians or double-blind trials, we found no statistically significant pooled benefits. We also found statistically significant pooled benefits when treatment was administered $30 \mathrm{~min}$ after ET and implantation phase. The effect of acupuncture administered at follicle phase and around ET on the CPR in women undergoing IVF was statistically significant.

Blinding of the physicians performing the ET was a potential source of bias. Our study showed bias between acupuncture and controls when combining all trials performed around the time of ET (fig. 2, 3). For the no intervention-controlled trials the RR descended gradually in the unblinded trials, trials blinded to physicians and double-blind trials (RR 1.54, 1.04 and 1.05, respectively). Comparing the acupuncture group with sham acupuncture controls when performed around the time of ET, the outcome was the same as the no intervention-controlled trials (RR 1.27, 0.98 and 0.95 , respectively).

There were some reasons for the distinct results of our systematic review. First, as mentioned, blinding of the physicians performing the ET was a potential source of bias. We separated the blinding and no blinding groups to decrease the risk of bias. Second, we used sh- am-controlled trials to rule out the possibility that acupuncture produces only psychological or psychosomatic effects [27]. Third, we concluded the effects of acupuncture at different time points. The times of acupuncture intervention were always another potential source of bias [8].

Women with previous IVF failure had lower clinical pregnancy outcomes than those undergoing treatment in their first cycle [36]. Patients should be included in the same IVF cycle in order to reduce a potential bias of baseline pregnancy rate. However, across all trials, the included patients were undergoing IVF or ICSI that included the first cycle or the repeated cycle. Many authors did not report how many previous cycles the patients underwent. Therefore we did not evaluate this in our design. In addition, the subgroup analysis of the review paper by Manheimer et al. [37] showed that such factors have not been shown to be strong predictors of pregnancy rate and none of these factors seemed to correlate well with the variability in baseline rate.

The efficacy of acupuncture given may vary in the included RCTs. High dosages of acupuncture could have a higher efficacy of acupuncture than the low dosage of acupuncture used in IVF $[38,39]$. However, the definitive efficient dosages are still unknown. Future research should consider the efficacy of dosage to reach an available outcome.

The protocol of many studies was as close as possible to the protocol used in the study of Paulus et al. [20]. Hence what the studies were doing was attempting to manifest the efficacy of performing around ET on the success rate in IVF. In fact, when only performed at one time point, acupuncture did not develop perfect function. Brenner [40] stated that this is a widespread issue with acupuncture trials as a one-size-fits-all protocol; these trials only give information on that protocol and not on the possibility of success with acupuncture. In the study of di Villahermosa et al. [31], acupuncture was performed on the 1st and 7th day of ovulation induction, on the day before ovarian puncture and on the day after ET. The study showed that the CPR in the acupuncture group was significantly higher than that in the control and sham groups. Our study inferred that acupuncture performed during controlled ovarian hyperstimulation and around ET would promote clinical pregnancy, and that performing acupuncture at $30 \mathrm{~min}$ after ET and implantation phase would have some effect on pregnancy outcomes.
Shen/Wu/Shu/Zhao/Gao 


\section{Conclusion and Future Research}

Our study showed that acupuncture increased pregnancy rates when performed at follicle phase and $25 \mathrm{~min}$ before and after ET or 30 min after ET and after the implantation phase in the IVF process. However, there was no statistically significant difference in women undergoing IVF between treatment performed only around the time of ET, follicle phase or implantation phase. The study of Zheng et al. [14] also showed that the pooled CPR result around the time of ET showed no significant differences between all acupuncture groups and the control groups. However, the CPR result of acupuncture performed around the time of controlled ovarian hyperstimulation showed a significant difference. The potential impact of acupuncture in the treatment of female infertility on the hypothalamic-pituitary-ovarian axis and on the uterus was significant, but the specific mechanism was still unknown [41]. Many medications such as oral contraceptive pills have no influence on increasing the pregnancy rate [42] and have some side effects, while acupuncture on patients undergoing IVF is a relatively simple procedure without side effects. Moreover, it was an inexpensive treatment for many couples affected by infer- tility $[10,43]$. Perhaps if more individualized acupuncture programs are used, more positive effects from acupuncture in IVF can be achieved [14]. The effects of acupuncture changed according to the endocrine system of patients, and so we should use acupuncture at different time points successively in the controlled ovarian hyperstimulation process according to individual characteristics. In addition, with some couples affected by infertility, lifestyle behavior also plays an essential role when pursuing assisted reproductive technology [44].

For future trials, the baseline rate of pregnancy, the dosage of acupuncture and individual characteristics should be considered. The protocol of efficient acupuncture should be well explored.

\section{Acknowledgments}

We are grateful to the members of the Department of Epidemiology and Biostatistics, School of Public Health, Tongji Medical College, Huazhong University of Science and Technology, who helped with the statistical analysis.

No specific funding was received for the study, but the Department of Obstetrics and Gynecology, Union Hospital, Tongji Medical College, Huazhong University of Science and Technology supported us throughout the study period and manuscript preparation.

\section{References}

$1 \mathrm{Ng} \mathrm{EH}$, So WS, Gao J, Wong YY, Ho PC: The role of acupuncture in the management of subfertility. Fertil Steril 2008;90:1-13.

2 Dalton-Brewer N, Gillott D, Atalla N, Menabawey $\mathrm{M}$, Wright $\mathrm{P}$, Thornhill A: Use of acupuncture before and after embryo transfer. Hum Fertil 2009;12:247.

3 Chen J, Liu LL, Cui W, Sun W: Effects of electroacupuncture on in vitro fertilization-embryo transfer (IVF-ET) of patients with poor ovarian response. Zhongguo Zhen Jiu 2009; 29:775-779.

4 Cavalla H: Acupuncture and electroauriculotherapy treatment for infertility. Med Acupunct 2010;22:49-52.

5 Sun L, Tao F, Hao J, Su P, Xu R, Liu F: Vaginal bleeding in early pregnancy and associations with physical, psychological and environmental factors among Chinese women: from the C-ABC cohort study. Gynecol Obstet invest 2012;73:330-336.

6 Balk J, Catov J, Horn B, Gecsi K, Wakim A: The relationship between perceived stress, acupuncture, and pregnancy rates among IVF patients: a pilot study. Complement Ther Clin Pract 2010;16:154-157.
7 Cakmak YO: Electroacupuncture reduces uterine artery blood flow impedance. Taiwan J Obstet Gynecol 2010;49:390, author reply 391.

8 Guo J, Li D, Zhang QF: Acupuncture intervention combined with assisted reproductive technology: its different effects at different time points during the in vitro fertilizationembryo transfer course (in Chinese). Zhong Xi Yi Jie He Xue Bao 2008;6:1211-1216.

$\checkmark 9$ Rashidi BH, Tehrani ES, Hamedani NA, Pirzadeh L: Effects of acupuncture on the outcome of in vitro fertilisation and intracytoplasmic sperm injection in women with polycystic ovarian syndrome. Acupunct Med 2013;31:151-156.

10 Craig LB, Criniti AR, Hansen KR, Marshall LA, Soules MR: Acupuncture lowers pregnancy rates when performed before and after embryo transfer. Fertil Steril 2007;88(suppl 1):S40.

11 Madaschi C, Braga DP, Figueira Rde C, Iaconelli A Jr, Borges E Jr: Effect of acupuncture on assisted reproduction treatment outcomes. Acupunct Med 2010;28:180-184.

12 El-Toukhy T, Sunkara SK, Khairy M, Dyer R, Khalaf Y, Coomarasamy A: A systematic review and meta-analysis of acupuncture in in vitro fertilisation. BJOG 2008;115:1203-1213.
13 Manheimer E, van der Windt D, Cheng K, Stafford K, Liu J, Tierney J, Lao L, Berman BM, Langenberg P, Bouter LM: The effects of acupuncture on rates of clinical pregnancy among women undergoing in vitro fertilization: a systematic review and meta-analysis. Hum Reprod Update 2013;0:1-10.

14 Zheng $\mathrm{CH}$, Zhang MM, Huang GY, Wang W: The role of acupuncture in assisted reproductive technology. Evid Based Complement Alternat Med 2012;2012:1-15.

15 El-Toukhy T, Khalaf Y: The impact of acupuncture on assisted reproductive technology outcome. Curr Opin Obstet Gynecol 2009;21: 240-246.

16 Stener-Victorin E: The pain-relieving effect of electro-acupuncture and conventional medical analgesic methods during oocyte retrieval: a systematic review of randomized controlled trials. Hum Reprod (Oxford) 2005;20:339349 .

17 Andersen D, Lossl K, Nyboe Andersen A, Furbringer J, Bach H, Simonsen J, Larsen EC: Acupuncture on the day of embryo transfer: a randomized controlled trial of 635 patients. Reprod Biomed Online 2010;21:366-372.
The Role of Acupuncture in in vitro Fertilization
Gynecol Obstet Invest 2015;79:1-12 DOI: $10.1159 / 000362231$ 
$\checkmark 18$ Domar AD, Meshay I, Kelliher J, Alper M, Powers RD: The impact of acupuncture on in vitro fertilization outcome. Fertil Steril 2009; 91:723-726.

$\checkmark 19$ Moy I, Milad MP, Barnes R, Confino E, Kazer $\mathrm{RR}$, Zhang X: Randomized controlled trial: effects of acupuncture on pregnancy rates in women undergoing in vitro fertilization. Fertil Steril 2011;95:583-587.

20 Paulus WE, Zhang M, Strehler E, El-Danasouri I, Sterzik K: Influence of acupuncture on the pregnancy rate in patients who undergo assisted reproduction therapy. Fertil Steril 2002;77:721-724.

-21 So EW, Ng EH, Wong YY, Lau EY, Yeung WS, Ho PC: A randomized double blind comparison of real and placebo acupuncture in IVF treatment. Hum Reprod 2009;24:341348.

22 Zhang M, Huang G, Lu F, El-Danasouri I, Sterzik K: Effect of acupuncture on rate of pregnancy among women undergoing embryo transfer. Zhongguo Zhen Jiu 2002;22: 507-509.

23 Zhang M, Huang G, Lu F, El-Danasouri I, Sterzik K: Effect of acupuncture on rate of pregnancy among women undergoing embryo transfer: a prospective randomized placebo controlled trial. Zhongguo Zhen Jiu 2003;23:3-5.

-24 Omodei U, Piccioni G, Tombesi S, Dordoni D, Fallo L, Ghilardi F: Effect of acupuncture on rates of pregnancy among women undergoing in vitro fertilization. Fertil Steril 2010; 94(suppl 1):S170.

-25 Benson MR, Elkind-Hirsch KE, Theall A, Fong K, Hogan RB, Scott RT: P-18: impact of acupuncture before and after embryo transfer on the outcome of in vitro fertilization cycles: a prospective single-blind randomized study. Fertil Steril 2006;86:S135.
26 Fratterelli JL, Leondires MR, Fong K, Theall A, Locatelli S, Scott RT: Laser acupuncture before and after embryo transfer improves art delivery rates: results of a prospective randomized double-blinded placebo controlled five-armed trial involving 1,000 patients. Fertil Steril 2008;90(suppl):S105.

27 Paulus WE, Zhang M, Strehler E, Seybold B, Sterzik K: Placebo-controlled trial of acupuncture effects in assisted reproduction therapy. Hum Reprod 2003;18:18-19.

28 Westergaard LG, Mao Q, Krogslund M, Sandrini S, Lenz S, Grinsted J: Acupuncture on the day of embryo transfer significantly improves the reproductive outcome in infertile women: a prospective, randomized trial. Fertil Steril 2006;85:1341-1346.

29 Cui W, Sun W, Liu L, Wen J: Effects of electroacupuncture on patients undergoing in vitro fertilization and embryo transplantation. Zhongguo Fu You Bao Jian 2006;22:34033405 .

30 Ho M, Huang LC, Chang YY, Chen HY, Chang WC, Yang TC, Tsai HD: Electroacupuncture reduces uterine artery blood flow impedance in infertile women. Taiwan J Obstet Gynecol 2009;48:148-151.

31 di Villahermosa DIM, dos Santos LG, Nogueira MB, Vilarino FL, Barbosa CP: Influence of acupuncture on the outcomes of in vitro fertilisation when embryo implantation has failed: a prospective randomised controlled clinical trial. Acupunct Med 2013;31:157161.

32 Smith C, Coyle M, Norman RJ: Influence of acupuncture stimulation on pregnancy rates for women undergoing embryo transfer. Fertil Steril 2006;85:1352-1358.

- 33 Dieterle S, Ying G, Hatzmann W, Neuer A: Effect of acupuncture on the outcome of in vitro fertilization and intracytoplasmic sperm injection: a randomized, prospective, controlled clinical study. Fertil Steril 2006;85: 1347-1351.

-34 Zhang R, Feng XJ, Guan Q, Cui W, Zheng Y, Sun W, Han JS: Increase of success rate for women undergoing embryo transfer by transcutaneous electrical acupoint stimulation: a prospective randomized placebo-controlled study. Fertil Steril 2011;96:912-916.
35 So EW, Ng EH, Wong YY, Yeung WS, Ho PC: Acupuncture for frozen-thawed embryo transfer cycles: a double-blind randomized controlled trial. Reprod Biomed Online 2010; 20:814-821.

36 Chiara R, Carolina B, Franco C: Effect of acupuncture on pregnancy rate in selected women undergoing IVF. Eur J Integr Med 2012;4: 52.

37 Manheimer E, Zhang G, Udoff L, Haramati A, Langenberg P, Berman BM, Bouter LM: Effects of acupuncture on rates of pregnancy and live birth among women undergoing in vitro fertilisation: systematic review and meta-analysis. BMJ 2008;336:545-549.

- 38 Humaidan P, Brock K, Bungum L, StenerVictorin E: Pain relief during oocyte retrieval - exploring the role of different frequencies of electro-acupuncture. Reprod Biomed Online 2006;13:120-125.

39 Anderson BJ, Rosenthal L: Acupuncture and IVF controversies. Fertil Steril 2007;87:1000.

40 Brenner Z: Acupuncture and IVF trial. Fertil Steril 2009;92:27, author reply 28.

41 Kim J, Shin KH, Na CS: Effect of acupuncture treatment on uterine motility and cyclooxygenase-2 expression in pregnant rats. Gynecol Obstet Invest 2000;50:225-230.

42 Bozdag G, Esinler I, Yarali H: Pretreatment with oral contraceptive pills does not influence the pregnancy rate in the long leuprolide acetate protocol. Gynecol Obstet Invest 2012; 73:53-57.

43 Rosenthal L, Anderson B: Acupuncture and in vitro fertilisation: recent research and clinical guidelines. J Chin Med 2007;84:28-35.

44 Domar AD, Conboy L, Denardo-Roney J, Rooney KL: Lifestyle behaviors in women undergoing in vitro fertilization: a prospective study. Fertil Steril 2012;97:697-701. 